\title{
Static friction on the fly: velocity depinning transitions of lubricants in motion
}

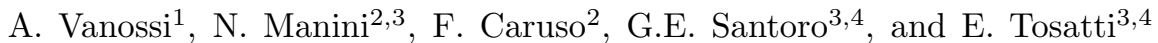 \\ ${ }^{1}$ CNR-INFM National Research Center S3 and Department of Physics, \\ University of Modena and Reggio Emilia, Via Campi 213/A, 41100 Modena, Italy \\ ${ }^{2}$ Dipartimento di Fisica and CNR-INFM, Università di Milano, Via Celoria 16, 20133 Milano, Italy \\ ${ }^{3}$ International School for Advanced Studies (SISSA) and CNR-INFM \\ Democritos National Simulation Center, Via Beirut 2-4, I-34014 Trieste, Italy \\ ${ }^{4}$ International Centre for Theoretical Physics (ICTP), P.O.Box 586, I-34014 Trieste, Italy
}

(Dated: October 29, 2018)

\begin{abstract}
The dragging velocity of a model solid lubricant confined between sliding periodic substrates exhibits a phase transition between two regimes, respectively with quantized and with continuous lubricant center-of-mass velocity. The transition, occurring for increasing external driving force $F_{\text {ext }}$ acting on the lubricant, displays a large hysteresis, and has the features of depinning transitions in static friction, only taking place on the fly. Although different in nature, this phenomenon appears isomorphic to a static Aubry depinning transition in a Frenkel-Kontorova model, the role of particles now taken by the moving kinks of the lubricant-substrate interface. We suggest a possible realization in $2 \mathrm{D}$ optical lattice experiments.
\end{abstract}

PACS numbers: 68.35.Af, 05.45.Yv, 62.25.+g, 62.20.Qp, 81.40.Pq, 46.55.+d

One of the current areas of development in the modern understanding of sliding friction is the depinning transition between substrate and slider in static friction [1]. A slider initially pinned on a substrate requires a finite force in order to move, whereas an unpinned one will move even under an infinitesimal force. It is generally known that ideal crystalline sliders commensurate with periodic substrates are always pinned, whereas incommensurate ones exhibit as a function of material parameters a sharp transition between a rigid, pinned state for soft sliders on strongly attractive substrates and a freely sliding state for hard sliders on weakly attractive perfect substrates. The standard paradigm for that transition [2] is the Aubry transition in the time-honored onedimensional (1D) Frenkel-Kontorova (FK) model [3, 4] . Once sliders are set into motion, however, one would generally expect no further rigidity or pinning to persist or to appear. It was thus rather surprising to find that simple systems like the 3-lengthscale driven 1D model of Ref. [5], inspired by the tribological problem of two sliding crystal surfaces with a thin solid lubricant layer in between, exhibits a novel kind of rigidity, now around a state of dynamical motion, where the lubricant centerof-mass $(\mathrm{CM})$ velocity is robustly locked onto quantized plateau values [6, 7, 8]. The dynamical pinning of the lubricant's motion onto a rigidly quantized relative velocity state has been understood in terms of kinks of the lubricant being set into motion by the shear due to the moving surfaces. This kind of kink dragging can be argued to represent a rather general mechanism, possibly at play also in more realistic two-dimensional situations.

One such situation could be realized e.g., by attempting to slide a rare gas physisorbed layer on a crystalline surface, by means of an external dragging agent, such as an AFM tip, or an optical tweezer, or a 2D optical lattice. In addition, the substrate could be oscillated in a Quartz
Crystal Microbalance (QCM), the inertial force acting as an (infinite wavelength) dragging agent. In either case, the external potential would in reality drag the solitons or discommensurations formed by the adsorbate with the substrate - it would drag the 2D Moiré patterns. While such a class of phenomena has not yet been explored, it is potentially quite interesting, and its extent and consequences deserve a full theoretical understanding. In this Letter we present a close analysis revealing that the lubricant velocity-pinned state shares many more of the characters typical of ordinary pinning in static friction, including hysteresis against a depinning external force $F_{\text {ext }}$, and a genuine depinning transition as a function of lubricant stiffness, that appears a dynamical isomorph to the Aubry transition of static friction.

Starting with a chain of harmonically interacting particles [9] (the lubricant layer), the Hamiltonian [6, 7]

$$
\begin{aligned}
H & =\sum_{i}\left[\frac{p_{i}^{2}}{2 m}+\frac{K}{2}\left(x_{i+1}-x_{i}-a_{0}\right)^{2}\right. \\
& \left.+\frac{U_{+}}{2} \cos \frac{2 \pi x_{i}}{a_{+}}+\frac{U_{-}}{2} \cos \frac{2 \pi\left(x_{i}-v_{\text {ext }} t\right)}{a_{-}}-F_{\text {ext }} x_{i}\right]
\end{aligned}
$$

contains the interaction with the two mutually sliding substrates of sinusoidal amplitudes $U_{+}$and $U_{-}$and periods $a_{+}$and $a_{-}$, different from the rest length $a_{0}$ of the springs, which sets a third length scale. Introducing dimensionless length ratios $r_{ \pm}=a_{ \pm} / a_{0}$ we assume $r_{-}>\max \left(r_{+}, r_{+}^{-1}\right)$, whereby the $(+)$ slider has the closest periodicity to the lubricant, the $(-)$ slider the furthest. To study depinning, we here apply a uniform external force $F_{\text {ext }}$ to all chain particles. The infinite chain size is managed - in the general incommensurate case - by means of periodic boundary conditions (PBC) and finite-size scaling [6, 8]. Sliding the substrates at constant relative velocity $v_{\text {ext }}$, the lubricant equations 
of motion are integrated by a standard Runge-Kutta algorithm, including a phenomenological viscous friction force $-2 \gamma\left(\dot{x}_{i}-\frac{1}{2} v_{\text {ext }}\right)=-2 \gamma\left(\dot{x}_{i}-v_{\mathrm{w}}\right)$ accounting for various sources of dissipation. As shown earlier [6, 7], for $F_{\text {ext }}=0$ and a wide range of parameters, the sliding chain reaches a dynamical stationary state characterized by a quantized plateau velocity

$$
v_{\mathrm{cm}} / v_{\text {ext }}=v_{\text {plateau }} / v_{\text {ext }} \equiv 1-r_{+}^{-1},
$$

depending solely on the incommensurability ratio $r_{+}$, independent of $r_{-}, K, \gamma, v_{\text {ext }}$, and even of $U_{-} / U_{+}$. In this quantized state of motion the array of topological solitons (kinks for $r_{+}>1$ or antikinks for $r_{+}<1$ ) formed by the chain with the least mismatched $(+)$ substrate is rigidly dragged at velocity $v_{\text {ext }}$ by the most mismatched (-) substrate. This dynamical pinning of the kink velocity to the moving $(-)$ slider suggests a more complete analogy to the static pinned state of particles in the standard FK model, where a finite force $F_{\text {ext }}>F_{c}^{\mathrm{FK}}$ is required to slide the chain over a periodic substrate. Depending on the ratio $\theta^{\mathrm{FK}}$ between the substrate and chain periodicities, static pinning in the FK model occurs for arbitrary $K$ for rational $\theta^{\mathrm{FK}}$, whereas irrational $\theta^{\mathrm{FK}}$ 's show a depinning (Aubry) transition taking place at a critical chain stiffness $K_{\text {Aubry }}$, beyond which free sliding is induced by arbitrarily small $F_{\text {ext }}, 3$, 4]. In the present slider-lubricant-slider problem, the kinks formed by the lubricant over the $(+)$ slider form a 1D lattice with an average distance $d_{\mathrm{k}}=a_{+} /\left(r_{+}-1\right)$, implying a kink coverage $\theta=a_{-} / d_{\mathrm{k}}=r_{-}\left(1-r_{+}^{-1}\right)$ of the $(-)$ substrate (for antikinks, $\theta<0$ ). If the kinks here play the role of the particles in the FK model, we should be able, depending on the relative commensurability $\theta$, to observe either indefinite pinning (i.e., quantized lubricant velocity) in the commensurate case, or else an Aubry-like depinning transition in the incommensurate case. We find that this is what happens, moreover with a clearly outlined isomorphism of the dynamical kink pinning problem to the static particle pinning of the FK model.

In order to highlight this deep similarity with the static case, beginning from a quantized-velocity state, we start off by studying the motion of the chain under the action of an adiabatically cycled external force $F_{\text {ext }}$. We select two prototypical kink coverages, one where the kink lattice is commensurate with the $(-)$ dragging slider $(\theta=1$, realized by $r_{+}=\phi, r_{-}=\phi^{2}$, with $\phi \equiv(1+\sqrt{5}) / 2$, the golden mean), and another incommensurate $(\theta=\phi$, obtained with $\left.r_{+}=\phi, r_{-}=\phi^{3}\right)$. The exactly quantized CM velocity plateau at $F_{\text {ext }}=0$ suggests null response to perturbations, so that any $0<F_{\text {ext }}<F_{c}^{+\uparrow}$ should have no effect whatsoever on $v_{\mathrm{cm}}$ ("incompressibility").

Figure 1 displays the result of cycling the external force $F_{\text {ext }}$ up and down in small steps, for $\theta=1$, $\gamma(K m)^{-1 / 2} \ll 1$ (underdamped regime), two different $v_{\text {ext }}$, and a chain stiffness $K$ within the velocity plateau. It displays a clear incompressibility and a hysteretic loop,

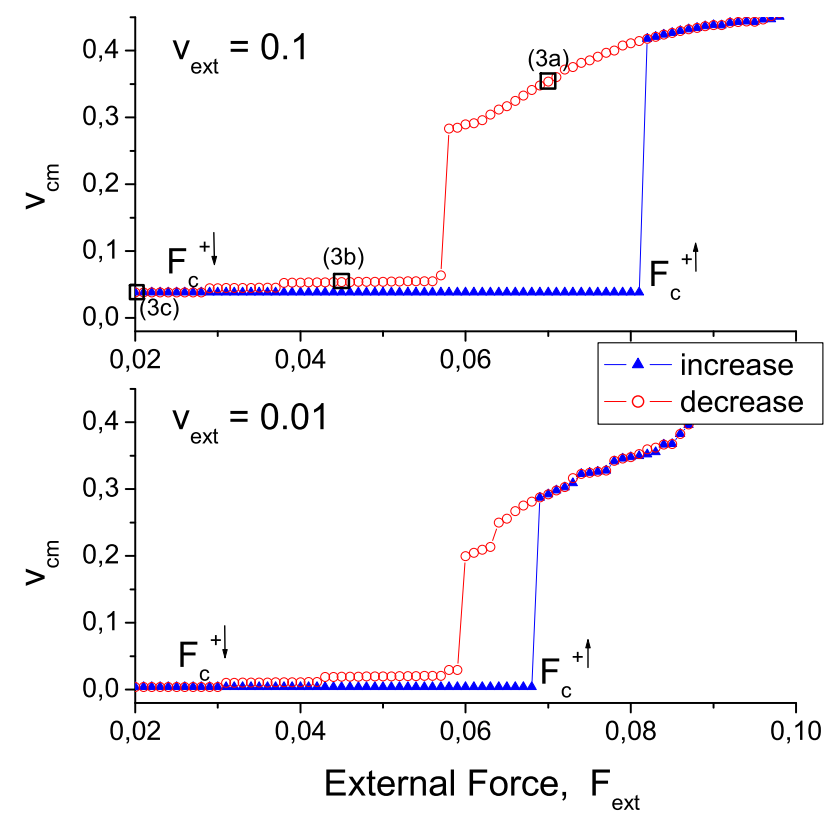

FIG. 1: (Color online) Hysteresis in the $v_{\mathrm{cm}}-F_{\text {ext }}$ characteristics for $\left(r_{+}, r_{-}\right)=\left(\phi, \phi^{2}\right)$. Throughout, our units are $m=1, a_{+}=1, F_{+}=2 \pi U_{+} / a_{+}=1, K$ is measured in $F_{+} / a_{+}, \gamma$ in $\sqrt{m F_{+} / a_{+}}$, velocities in $\sqrt{a_{+} F_{+} / m}$, and we choose $F_{-}=2 \pi U_{-} / a_{-}=F_{+}$. [8] The behavior is shown for fast $\left(v_{\text {ext }}=0.1\right.$, upper panel) and slow $\left(v_{\text {ext }}=0.01\right.$, lower panel) drive. Adiabatic increase and decrease of $F_{\text {ext }}$ is denoted by triangles and circles, respectively. A characteristic multi-step feature appears when decreasing adiabatically $F_{\text {ext }}$. Here $\gamma=0.1$ and $K=4$.

analogous to the depinning transition in static friction [1], and in the FK model [3]. For increasing $F_{\text {ext }}$ the lubricant average velocity $v_{\mathrm{cm}}$ is discontinuous at $F_{c}^{+\uparrow}$, with a dynamical depinning, a finite jump $\Delta v$ and a bistable behavior. When $F_{\text {ext }}$ is decreased back, the depinned state survives down to $F_{c}^{+\downarrow}<F_{c}^{+\uparrow}$, where quantized sliding is retrieved, as shown in Fig. 1. The hysteretic behavior observed under the application of a negative $F_{\text {ext }}$ is similar to that for $F_{\text {ext }}>0$, with corresponding $F_{c}^{-\downarrow}<F_{c}^{-\uparrow}<0$. Viewing the kinks of our model as the particles of a standard FK model, the $\theta=1$ case is isomorphic to the fully commensurate $\theta^{\mathrm{FK}}=1 \mathrm{FK}$ static case, where pinning holds for all $K$ 's. Confirming that, when we counterbalance the average frictional force $F_{\mathrm{w}}=-2 \gamma\left(v_{\text {plateau }}-v_{\mathrm{w}}\right)$ with an equal and opposite external force $F_{\text {ext }}=-F_{\mathrm{w}}$ such that no net force acts on the kink lattice, we find that the plateau extends out to arbitrarily large $K$, see Fig. 2(a). The observed phenomenology is quite generic: in suitable parameters ranges we find similar hysteretic plateaus for all values of $r_{+}$and $r_{-}$investigated. Moreover, different choices of $r_{-}=\theta /\left(1-r_{+}^{-1}\right)$, with rational $\theta \neq 1$ lead to plateaus which also extend to arbitrarily large $K$. A generic rational $\theta>1$ implies defects in the perfectly commensurate $(\theta=1)$ lattice of kinks, in the form of kinks of the kink lattice - hierarchical excita- 


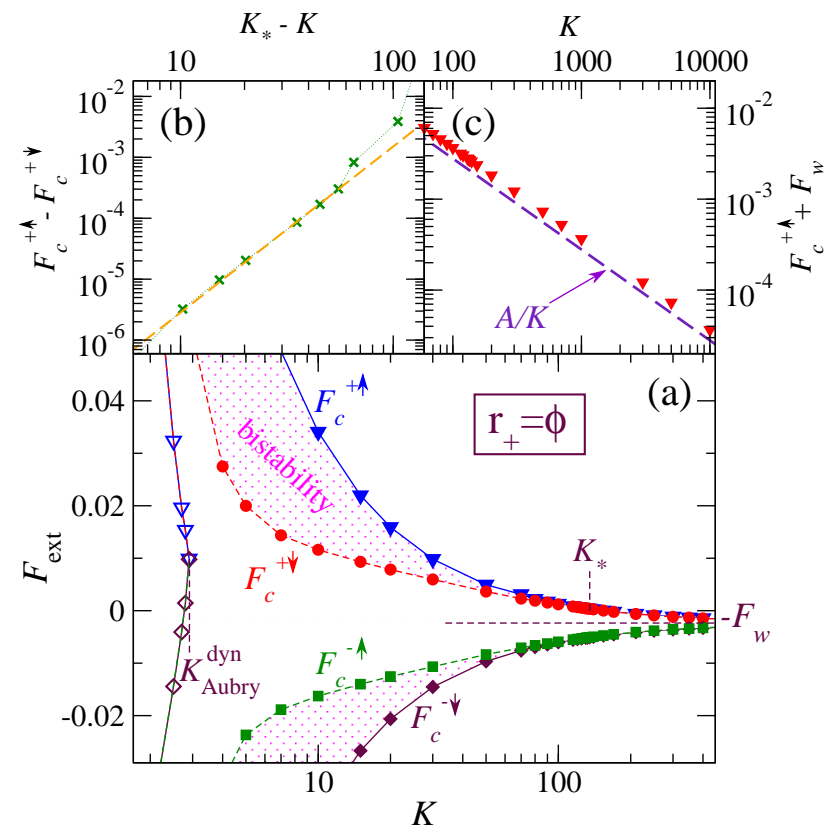

FIG. 2: (Color online) (a) The critical depinning $\left(F_{c}^{+\uparrow}, F_{c}^{-\downarrow}\right)$ and re-pinning $\left(F_{c}^{+\downarrow}, F_{c}^{-\uparrow}\right)$ forces, as functions of the chain stiffness $K$, for $\left(r_{+}, r_{-}\right)=\left(\phi, \phi^{2}\right)$ (i.e. commensurate $\theta=1$, solid symbols, pinning extending to infinitely large $K$ ) and for $\left(r_{+}, r_{-}\right)=\left(\phi, \phi^{3}\right)$ (i.e. incommensurate $\theta=\phi$, open symbols). In all calculations $v_{\text {ext }}=0.1, \gamma=0.1$. For $\theta=1$ : (b) the width $F_{c}^{+\uparrow}-F_{c}^{+\downarrow}$ of the bistability region as a function of $\left(K_{*}-K\right)$ (crosses), compared to a fitted power-law $F_{c}^{+\uparrow}-$ $F_{c}^{+\downarrow}=B\left(K_{*}-K\right)^{\alpha}$, with $\alpha \simeq 2.7, K_{*} \simeq 135$; (c) the large- $K$ depinning force, compared to the expected power-law $\left(F_{c}^{+\uparrow}+\right.$ $\left.F_{\mathrm{w}}\right)=A K^{-1}$, Eq. (3).

tions, kinks of kinks - which, being commensurate with (-) substrate, resist depinning for arbitrarily large $K$.

On the contrary, we expect - and find, see Fig. 2(a), for $\theta=\phi$ - that irrational $\theta$ should be associated with a genuine Aubry transition on the fly of the incommensurate lattice of kinks, with the plateau disappearing at $K_{\text {Aubry }}^{\text {dyn }}$. The transition takes place at a finite $K=K_{\text {Aubry }}^{\text {dyn }}$, where the width $F_{c}^{+\uparrow}-F_{c}^{-\downarrow}$ of the velocity plateau as a function of $F_{\text {ext }}$ shrinks to zero. Of course, we expect $K_{\text {Aubry }}^{\text {dyn }}$ to be a complicated function of the original model parameters, generally not coincident with the value characteristic of the FK model for $\theta^{\mathrm{FK}}=\theta$.

The pinning force $\left(F_{c}^{+\uparrow}+F_{\mathrm{w}}\right)$ decreases like $K^{-1}$ for large $K$, see Fig. 2(c). In this limit, the kink lattice becomes increasingly faint, since the corresponding particle-density modulation amplitude drops as $K^{-1}$. Quantitatively, the small displacements of the particles away from ideal lattice positions $x_{i}=i a_{0}$ due to interaction with the $(+)$ potential are described in terms of a hull function, whose explicit expression is known [10] for large $K \gg F_{+} / a_{+}$(with $\left.F_{+}=2 \pi U_{+} / a_{+}\right)$. These displaced positions, once substituted into the $U_{-}$term of Eq. (1), yield a sinusoidal oscillation as a function

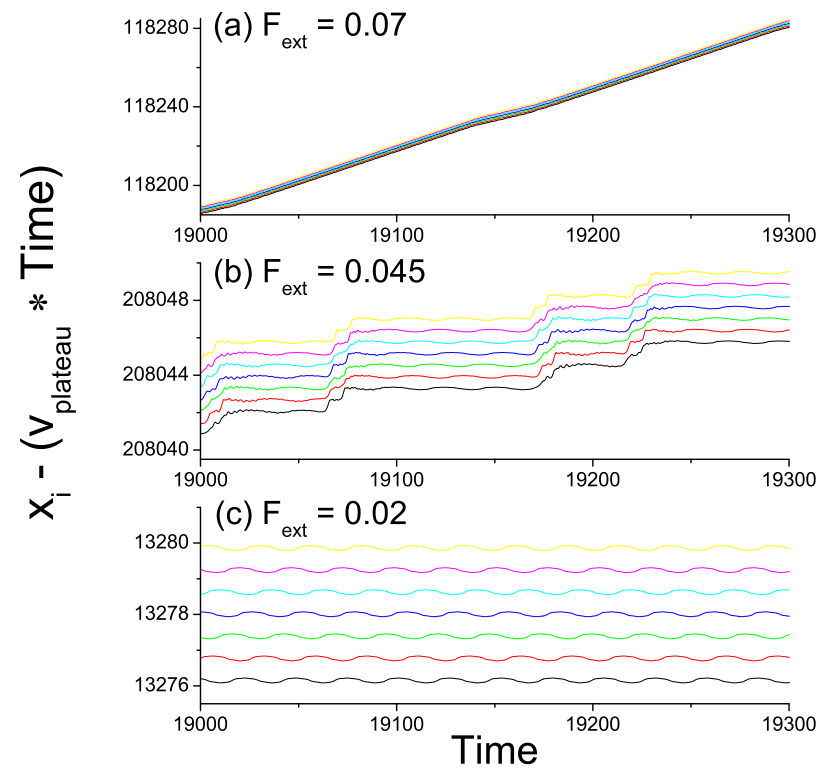

FIG. 3: (Color online) Time evolution of the positions $x_{i}-$ $v_{\text {plateau }} \cdot t$ of 7 contiguous chain particles. $t$ is measured in units of $\sqrt{m a_{+} / F_{+}}$. The plots refer to three dynamical regimes observed along the adiabatic decrease of $F_{\text {ext }}$, at the points indicated by (3a)-(3c) in Fig. 1 (a) free sliding at $F_{\text {ext }}=$ 0.07 , (b) dynamic stick-slip at $F_{\text {ext }}=0.045$, and (c) quantized sliding state at $F_{\text {ext }}=0.02$. Same parameters as in Fig. 1] with $v_{\text {ext }}=0.1$.

of the translation $v_{\text {ext }} t$ of the $(-)$ substrate relative to the kink lattice. This energy oscillation corresponds to a force whose maximum amplitude equals the minimum force that must be applied to the chain to have the kinks overcome the barrier and initiate sliding. This critical force per particle amounts to

$$
F_{c}^{+}+F_{w}=\frac{A}{K}+O\left(K^{-2}\right), \quad A=\frac{\pi}{8} \frac{r_{+}-1}{\sin ^{2} \frac{\pi}{r_{+}}} \frac{F_{+} F_{-}}{a_{+}},
$$

and is drawn in Fig. 2(c) for comparison. The $\simeq 20 \%$ discrepancy with the observed $F_{c}^{+}$is due to the neglect of the displacements induced by the $(-)$ potential, which enhance the kinks amplitude in this $\theta=1$ geometry.

The self-pinning at the $(-)$ minima is also at the root of the bistability found at moderate $K$ : self-enhanced trapped kinks resist to a large $F_{\text {ext }}$ up to $F_{c}^{+\uparrow}$, but as soon as kinks wash away in the large- $F_{\text {ext }}$ unpinned sliding state, $F_{\text {ext }}$ must be reduced to smaller $F_{c}^{+\downarrow}$ before kinks reconstruct and then re-pin. The transition boundaries $F_{c}^{+\uparrow}, F_{c}^{+\downarrow}$, and $\Delta v$ are nontrivial functions of the parameters. In particular, as shown in Fig. 2(a), for large $K$ the hysteretic behavior extends up to $K=K_{*} \simeq 135$, see power-law fit in Fig. 2(b). For $K \geq K_{*}, F_{c}^{+\uparrow} \equiv F_{c}^{+\downarrow}=F_{c}^{+}$, and the depinning transition is continuous, characterized by a critical behavior $\left(v_{\mathrm{cm}}-v_{\text {plateau }}\right) \propto\left(F_{\text {ext }}-F_{c}^{+}\right)^{1 / 2}$. On approaching the critical point $K_{*}$, the jump $\Delta v$ characterizing the 


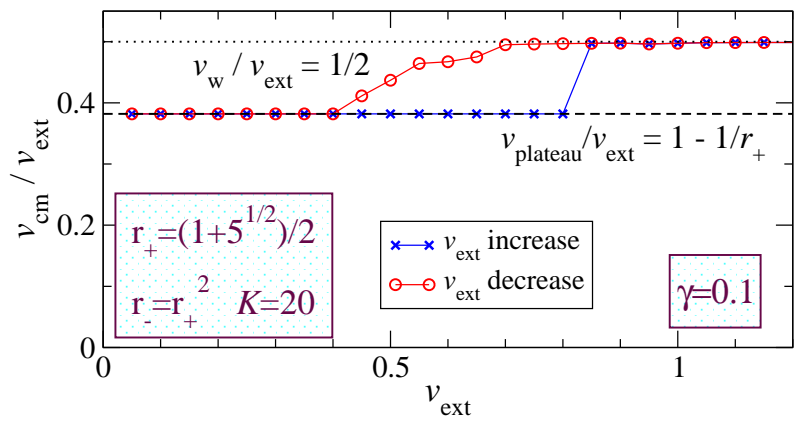

FIG. 4: (Color online) Hysteresis, as $v_{\text {ext }}$ is cycled, in the underdamped regime.

first-order depinning transition vanishes, and a continuous transition line originates there. In the moderate- $K$ hysteretic regime we find that, in a wide range of parameters, depinning occurs through a discontinuous jump to a quasi-free sliding regime $F_{\text {ext }}>F_{c}^{+\uparrow}$, see Fig. $3(a)$, characterized by aperiodic single-particle motion, superposed to a drift approaching the free-particle limit velocity $F_{\text {ext }} /(2 \gamma)$. The reverse route, upon decreasing $F_{\text {ext }}$ to $F_{c}^{+\downarrow}$, yields some insight in the re-pinning mechanism. Depending on the values of $K$ and $v_{\text {ext }}$, dynamical repinning to the plateau-state takes place through a variety of mechanisms, from intermittencies with a well defined periodicity, to more chaotic and irregular jumps. Despite that variety, for intermediate $K$ we consistently observe one or more multi-step sliding regimes as $F_{\text {ext }}$ decreases within the $\left[F_{c}^{+\downarrow}, F_{c}^{+\uparrow}\right]$ hysteresis window, consistently with the results known for the sine-Gordon [1], and FK [12] models. Our intermediate- $F_{\text {ext }}$ regime, illustrated in Fig. 3(b), is reminiscent of intermittencies representing a dynamic stick-slip occurring on a local scale, clearly seen by plotting the particle trajectories in the reference frame which slides at $v_{\text {plateau }}$. In fact, detailed global analysis of this intermediate- $F_{\text {ext }}$ dynamics, based on simulations with $r_{+}=1+\epsilon$ (with $\left.\epsilon \ll 1\right)$ where kinks are well separated, shows that the kinks pin to the (-) lattice along most of the chain, with a few kink-antikink defects travelling along the chain. These defective sections move along the chain at a characteristic speed depending on the model parameters. The nature of these de-pinning mobile defects is reminiscent of those found in the ordinary FK model [3]. Depending on $K$ and $F_{\text {ext }}$, single- or multiple-kink defects arise: transitions between intermediate phases characterized by different numbers of travelling-kink defects are visible in Fig. 1.

In practice, the experimental realization of an equal force acting on each particle could be provided, for instance, by the inertial forces in a QCM experiment. In addition, the hysteretic depinning and re-pinning occurs even when different tunable parameters are varied adiabatically, for instance the driving velocity $v_{\text {ext }}$, see Fig. 4, as long as we are in the underdamped limit [13]. Thus, in a concrete lab experiment, cycling quantities such as $v_{\text {ext }}$ should lead to leaving/recovering the plateau state, similar to cycling $F_{\text {ext }}$. As for the dragging agent, the typical force that a laser provides in an optical lattice of alkali atoms is $F \approx 10^{-9} \mathrm{pN} 14$. This is very small, but we checked that our phenomenology still survives even when $F_{-}$is orders of magnitude smaller than $F_{+}$. Larger (inertial) forces, $m \omega^{2} a \sim 10^{-5} \mathrm{pN}$ per adsorbate atom, are accessible in a standard QCM oscillating at $\omega / 2 \pi \sim 15$ $\mathrm{MHz}$ with amplitudes $a \sim 100 \AA$. The sinusoidal variation of the dragging velocity occurs here between 0 and $\pm 1 \mathrm{~m} / \mathrm{s}$ on a much longer time-scale than the typical adsorbate vibrational frequencies $(\sim 1 \mathrm{THz})$. The inertial dragging force couples to the local density fluctuations, and should drag the solitons exactly like a commensurate $(-)$ slider would do.

As for thermal effects, they favor unpinning, but as long as $k_{B} T$ is much smaller than the cost $\Delta$ for the creation of the relevant depinning defects, the physics does not change much, as shown by numerical simulations of similar models. [15]

Our overall picture confirms a striking similarity of the dynamical kink-depinning transition of a lubricant under shear to the usual depinning in static friction, and in the static FK model, to which the large- $K$ theory of Eq. (3) establishes a quantitative correspondence. These findings should also be amenable to experimental verification.

This research was partially supported by PRRIITT (Regione Emilia Romagna), Net-Lab "Surfaces \& Coatings for Advanced Mechanics and Nanomechanics" (SUP\&RMAN) and by MIUR PRIN 2006022847.

[1] S. M. Rubinstein et al., Nature 430, 1005 (2004).

[2] K. Shinjo, M. Hirano, Surf. Sci. 283, 473 (1993).

[3] O. M. Braun and Yu. S. Kivshar, The Frenkel-Kontorova Model: Concepts, Methods, and Applications (SpringerVerlag, Berlin, 2004).

[4] M. Peyrard and S. Aubry, J. Phys. C: Solid State Phys. 16, 1593 (1983).

[5] O. M. Braun et al., Phys. Rev. Lett. 95, 026102 (2005).

[6] A. Vanossi et al., Phys. Rev. Lett. 97, 056101 (2006).

[7] G. E. Santoro et al., Surf. Sci. 600, 2726 (2006).

[8] N. Manini et al., J. Phys.: Condens. Matter, 19, 305016 (2007).

[9] The choice of a harmonic interaction is not crucial: simulations with Morse-type and Lennard-Jones potentials also reveal the ubiquity of the observed phenomenology.

[10] T. S. van Erp et al., Phys. Rev. B 60, 6522 (1999).

[11] J. C. Ariyasu and A. R. Bishop, Phys. Rev. B 35, 3207 (1987).

[12] O. M. Braun et al., Phys. Rev. Lett. 79, 3692 (1997).

[13] In the overdamped limit, when the viscous rate $\gamma / m$ is larger than the characteristic frequencies of the kinklattice, the dynamical depinning turns second order.

[14] O. Mandel et al., Nature 425, 937 (2003).

[15] O. M. Braun et al., Phys. Rev. E 55, 3598 (1997). 